\title{
Design da informação: Mapeamento de informação e diagramas na representação de um projeto de pesquisa
}

Reinaldo Pereira de Moraes é Mestre em Design pela Universidade Federal do Paraná (2011), Especialista em Análise e Projeto de Sistemas Orientados a Objetos pela Universidade Federal do Paraná (2008), Especialista em Educação a Distância pela Universidade Cruzeiro do Sul-SP (2016), Especialista em Tecnologias Educacionais pela UNISOCIESC-SC (2016), Tecnólogo em Informática pela Universidade Tecnológica Federal do Paraná (2007) e Bacharel em Design pela Universidade Tecnológica Federal do Paraná (2019). Como professor de Ensino Superior na Faculdade Bagozzi em Curitiba-PR, ministra aulas e orienta alunos no desenvolvimento de projetos. $\mathrm{Na}$ área educacional, tem interesse no Estudo de Metodologias Educacionais. $\mathrm{Na}$ área da computação, tem interesse em Engenharia de Software. Na área do Design, tem em interesse em Avaliação de Interfaces, Estudo da Interação Humano-Computador, Ergonomia e Processos de Desenvolvimento em Design. <moraesreinaldo@yahoo.com.br> ORCID: 0000-0003-2193-653X
Resumo Este artigo apresenta, sob o ponto de vista do Design da Informação, uma análise comparativa a partir da representação gráfica de um projeto de pesquisa através do mapeamento de informação de Robert Horn (1993) e das ferramentas gráficas de Yvonne Hansen (1999). O objetivo deste artigo é comparar a representação de um projeto de pesquisa a partir do estudo das ferramentas propostas por estes autores. A metodologia adotada se baseia na pesquisa bibliográfica como principal procedimento técnico e a partir desta prática se propõe a analisar comparativamente as duas ferramentas trabalhadas. Como resultados, notou-se que o mapeamento de informação de Horn não é eficiente na fase inicial do estudo do problema, mas é útil quando é necessário organizar as informações; e que as ferramentas gráficas de Hansen podem ser usadas no processo inicial de especificação de um problema porque são eficientes na clarificação de ideias e compreensão de conceitos.

Palavras chave Design da informação, Mapeamento de informação, Diagramas de representação. 


\title{
Information Design: Information Mapping and Diagrams in the Representation of a Reseach Project
}

\begin{abstract}
This paper presents, from the point of view of Information Design, a comparative analysis from the graphic representation of a research project through the mapping of information by Robert Horn (1993) and the graphic tools of Yvonne Hansen (1999). The objective of this paper is to compare the representation of a research project from the study of the tools proposed by these authors. The methodology adopted is based on bibliographical research as the main technical procedure and from this practice it is proposed to analyze comparatively the two tools. As results, it was noted that Horn's information mapping is not efficient in the initial phase of problem study but it is useful when it is necessary to organize information; and that Hansen's graphic tools can be used in the initial process of specifying a problem because they are efficient in clarifying ideas and understanding concepts.
\end{abstract}

Keywords Information design, Mapping of information, Representation diagrams. 


\section{Introdução}

Para Silva e Menezes (2000, p. 85), “o projeto de pesquisa é um documento que tem por finalidade antever e metodizar as etapas operacionais de um trabalho de pesquisa". Na prática, a definição do projeto de pesquisa envolve muitos questionamentos referentes à originalidade e à relevância do projeto para a área de conhecimento estudada, bem como a adequação do projeto à capacidade intelectual do pesquisador. Além disso, deve-se também questionar sobre as possibilidades de tempo e recursos financeiros, além da existência de materiais ou equipamentos necessários para a realização da pesquisa. Questiona-se também a respeito do tempo disponível para se chegar a alguma descoberta, além de contextualizar o problema ambiental, política, social, economicamente para que se tenha a percepção clara do que está sendo estudado.

Todas estas informações poderiam ser descritas por meio de texto, respondendo e esclarecendo sobre cada questionamento e seus desdobramentos. E de fato, assim é feito. Contudo, para facilitar o entendimento de quem lê, ou para ajudar o pesquisador a entender o seu projeto de pesquisa, mesmo antes de ser descrito em palavras, é possível a elaboração de representações gráficas a fim de transmitir as informações necessárias. $O$ uso de diagramas e representações para esclarecer conceitos não é recente. Conforme Hansen (1999), profissionais e especialistas em música, coreografia, química, geometria, estatística, geografia, cartografia e geologia desenvolveram símbolos não verbais para esclarecer suas ideias e descobertas.

Conforme Archela (1999, p. 7), "a expressão da linguagem visual dispõe de meios diferentes daqueles utilizados pela linguagem verbal, mas o encaminhamento do pensamento, para chegar a um resultado idêntico a comunicação é a mesma: um sistema lógico de raciocínio". Entretanto, apenas o encadeamento lógico não é suficiente para o entendimento, pois, "a representação gráfica é um instrumento de reflexão que permite analisar um problema através de questões pertinentes e descobrir informações, e demanda dois tempos de percepção: 1o) que coisas os signos simplificam? 20) quais são as relações entre as coisas?" (ARCHELA, 1999, p. 9).

Por meio deste artigo, pretende-se estudar a ferramenta de mapeamento de informação de Robert Horn (1993) e as ferramentas gráficas de Yvonne Hansen (1999) a fim de comparar sua capacidade de representação de um projeto de pesquisa.

\section{Metodologia}

A pesquisa realizada é de natureza teórica com uma abordagem qualitativa a fim de cumprir com seu objetivo explicativo e descritivo adotando a revisão bibliográfica como procedimento técnico. A partir da definição do objeto a ser estudado, um projeto de pesquisa, e com base na fundamentação teórica sobre representação gráfica através do mapeamento de informação de Robert Horn e das ferramentas gráficas de Yvonne Hansen, 
executa-se a aplicação prática destas ferramentas na representação gráfica do projeto de pesquisa. A partir deste procedimento, desenvolve-se uma análise comparativa entre as duas ferramentas trabalhadas.

\section{Mapeamento de Informação de Robert Horn}

De 1963 a 1965, Robert Horn desenvolveu um trabalho sobre a escrita estruturada, chamado de mapeamento de informação como uma pesquisa acadêmica, cujo interesse era desenvolver materiais didáticos de conteúdos que fossem mais fáceis e rápidos para se aprender (HORN, 1999). Seu trabalho está divido em três grandes partes: análise do conteúdo, integração e síntese do ciclo de vida, e sequenciamento e formato (HORN, 1992).

\section{Análise do conteúdo}

O foco desta parte do método é conceber uma taxonomia e critérios para o novo conjunto das menores unidades práticas de significado para a escrita de documentos. Esta modularidade tem o objetivo de ensinar os escritores, de maneira fácil e com grande uniformidade, a escolher sentenças e diagramas dentro de uma taxonomia facilmente entendida (HORN, 1999).

A partir deste processo, surgiu uma nova unidade chamada de "bloco de informação". Embora o mapeamento de informação não tenha sido o primeiro a usar o conceito de modularidade, foi o primeiro a definir e desenvolver um conceito modular preciso, o bloco de informação, que é firmemente fundamentado na taxonomia dos tipos de informação (HORN, 1999). Os blocos de informação (Horn, 1992), são as unidades básicas de assuntos na escrita estruturada. Eles substituem os parágrafos como unidades fundamentais de análise e de apresentação. Eles são compostos de uma ou mais sentenças e/ou diagramas sobre o assunto delimitado, normalmente não mais que nove sentenças e são claramente identificados por um rótulo. Normalmente fazem parte de uma ampla estrutura de organização chamada de mapa de informação que pode ser definido como uma coleção de um a nove blocos, todos relacionados a um assunto específico. Para Horn (1993), existe algo fundamental sobre a taxonomia gerada, pois este esquema de blocos de informação é capaz de triar $80 \%$ ou mais do conteúdo dos assuntos abordados, portanto, pode-se dizer que o método captura e classifica a essência das sentenças.

\section{Integração e Síntese do Ciclo de Vida}

A análise do conteúdo é, eventualmente, integrada com vários métodos de planejamento do ciclo de vida. Segundo o autor, isso ocorre, principalmente para projetos que são complexos (HORN, 1993). A integração do ciclo de vida foi elaborada para facilitar projetos de escrita de documentos 
em todos os níveis de detalhes e tamanhos, desde um memorando até projetos de documentos maiores e mais complexos encontrados nas indústrias e academias (HORN, 1992). A integração e síntese do ciclo de vida são importantes e começam com uma definição inicial da necessidade de gerenciamento da informação, para o sequenciamento e escolha final do meio de apresentação e formato (HORN, 1993). Fazer a análise do conteúdo, adequadamente dividida e marcada, contribui para a eficiência ao longo do caminho do ciclo de vida do documento (HORN, 1992). Isso ajuda a especificar quais informações estão faltando em um dado momento do processo (HORN, 1993). Para ele, saber o que você não sabe é uma importante vantagem.

\section{Sequenciamento e Formato}

Horn (1993) define o sequenciamento e formatação como a habilidade de especificar o sequenciamento precisamente e a habilidade de conceber muitos formatos para apresentação da informação complementar para o sistema de análise de conteúdo, respectivamente. Para o autor, o desafio do sequenciamento tem sido especificar padrões na maneira como um escritor ou editor pode se comunicar precisamente com outro sobre exatamente quais blocos de informação estarão em exatamente que ordem na apresentação final do material.

O método provê modelos de sequenciamento básico e facilita a comunicação de maneira precisa sobre o padrão de sequenciamento (HORN, 1992). $O$ autor ainda acrescenta que os mapas de informação devem guiar o leitor da mesma forma que os mapas geográficos fazem, e que a habilidade de mostrar relacionamentos e guiar o usuário rapidamente para lugares relevantes é a chave para entender a metáfora do nome dos mapas de informação.

Compreender isso é importante porque os leitores estão envolvidos com todas as informações que eles precisam processar todos os dias em situações de negócios, ciências e tecnologia, envolvendo a exploração de documentos a fim de encontrar partes importantes que lhes interessam (HORN, 1999). 0 autor sugere o uso de gráficos próximos ao texto a que se referem e não em outros locais. Para ele as informações em formato de gráficos, diagramas e ilustrações devem ser preferidos em vez de textos. A integração de gráficos e palavras permite uma exploração e leitura mais rápida de documentos porque eles não precisam procurar por aquilo que lhes interessa no documento inteiro (HORN, 1999). Para o autor, a linguagem visual se tornará mais importante que seus antecessores e, a linguagem visual combinada com o design de informação se tornará o maior método técnico no campo da documentação.

\section{A Apresentação Gráfica de um Mapa de Informação}

A Figura 1 apresenta uma das formas como os blocos de informação podem ser organizados, rotulados e dispostos a fim de facilitar a leitura do documento. 


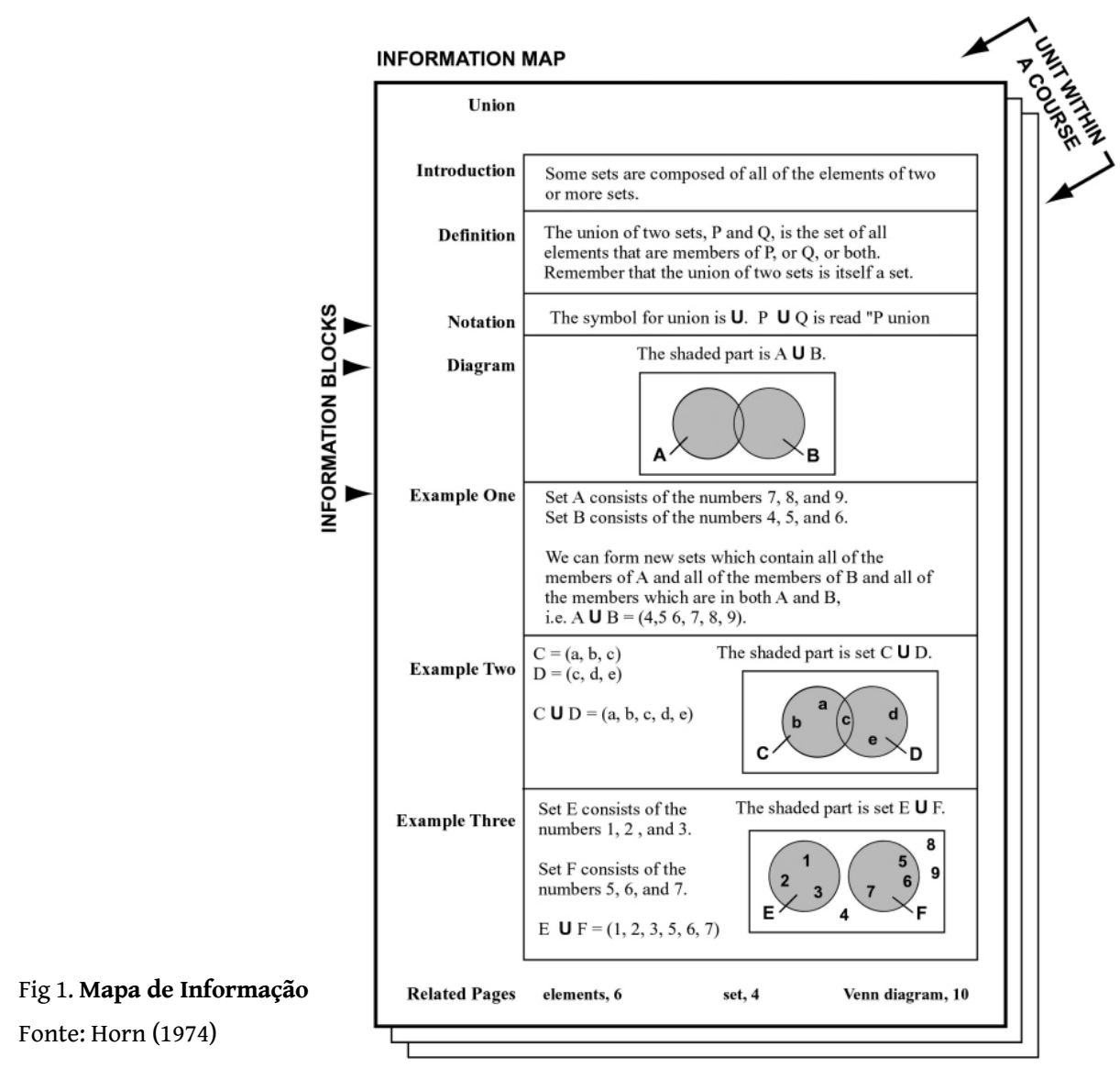

Dentro dos blocos de informação devem sem escritos os parágrafos com a síntese do conteúdo, com diagramas, imagens e outras formas de representações que sejam necessárias. Percebe-se neste exemplo a presença de apenas seis blocos adjacentes. Poderiam ser utilizados outros blocos (até nove) que não fossem necessariamente adjacentes.

\section{Ferramentas Gráficas de Yvonne Hansen}

\section{Aquisição e Visualização da Informação}

Conforme Hansen (1999) a informação chega aos leitores de três fontes gerais: externa, interna e a combinação de externa e interna. A fonte externa é o mundo observável, tudo aquilo que é visto e percebido, como, por exemplo, textos impressos. A fonte interna diz respeito às ideias, visualização e percepção de cada um. A terceira fonte de informação dá origem a novas combinações de conhecimento e informações.

As palavras são poderosas o suficiente para comunicar quando são proferidas no tom certo e no tempo certo. As palavras não são o único e 
primário sentido da comunicação da informação, e os pensamentos e ideias não são formados primeiro como palavras e sentenças (HANSEN,1999). Sobre o processo de comunicação, a autora considera importante que o designer de informação esteja ciente de como funciona o processo de pensamento interno, porque ele influencia no processo de comunicação; e afirma que as palavras podem impedir o entendimento, porque elas são frequentemente inadequadas de acordo com a experiência de sobrecarga de informação. Entretanto, para ela, palavras e conceitos que representam ideias podem ser exibidas e armazenadas graficamente, em um mapa da mente. Para amparar a ideia da representação gráfica dos conceitos, a autora argumenta que, de alguma forma, o cérebro sabe que existe algo de valor no que conscientemente é percebido como aleatoriedade e desordem. Assim, a vantagem de usar gráficos junto com palavras e frases é estar apto para ver relacionamentos e estrutura entre os dados que são obscuros em uma situação de texto. Além disso, as informações de fonte interna também precisam ser gerenciadas (HANSEN, 1999). Neste sentido, embora não exista uma linguagem formalizada para capturar ideias criativas e sintetizar os pensamentos exploratórios, adicionar imagens para palavras, de alguma forma altera a maneira como um objeto ou ideia são percebidos (HANSEN, 1999). A este respeito, a autora acrescenta que simples formas gráficas podem representar a cognição pré-verbal e dar forma concreta a ela. Os gráficos conceituais e imagens têm sido usados como apoio a textos ou para ilustrar dados numéricos. Hansen (1999), entretanto, defende que eles podem adicionar uma perspectiva visual para a descrição verbal e revelar conexões não aparentes quando apenas palavras são usadas. Por isso, propõe ferramentas gráficas que possam expressar ideias e conceitos de forma mais eficiente.

\section{As Ferramentas Gráficas}

A ferramenta proposta por Hansen (1999) é composta por seis ferramentas gráficas, que possuem formas diferentes: círculos ou figuras curvadas, áreas com cantos arredondados ou ângulo reto, triângulos, linhas, pontos e rabiscos. Nas figuras 2 a 7, são exemplificadas algumas destas representações e como elas podem ser utilizadas.

Círculos ou figuras curvadas (figura 2) podem representar a inteireza; um elemento; um sistema ou subsistema; um conceito; possibilidade às vezes não ainda claramente definida ou rotulada; e são usados para fechar uma área; definir uma fronteira; alocar elementos como internos ou externos, enquanto que Áreas com ângulo reto (figura 3) podem representar um receptáculo de informações; fatos; políticas; exibições ou modelos, e são usados para conter textos; descrever um relacionamento entre elementos e é colocado entre os elementos e Áreas de cantos arredondados (figura 3) representam uma condição; um local; ambiente contexto, e são usadas para conter ou segurar um elemento; sistema; conceito e indicar um local (HANSEN, 1999). 


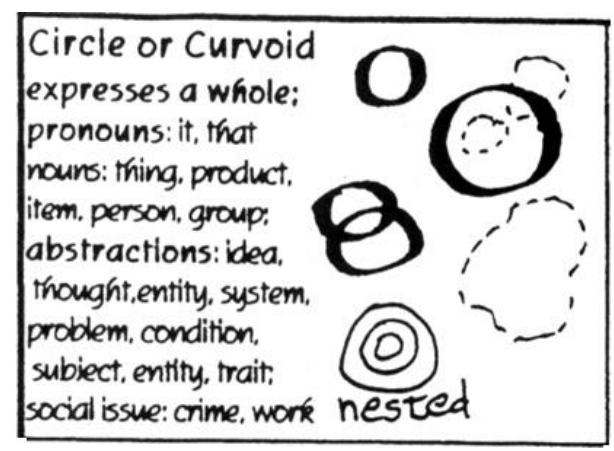

Fig 2. Círculos ou figuras curvadas

Fonte: Hansen (1999)

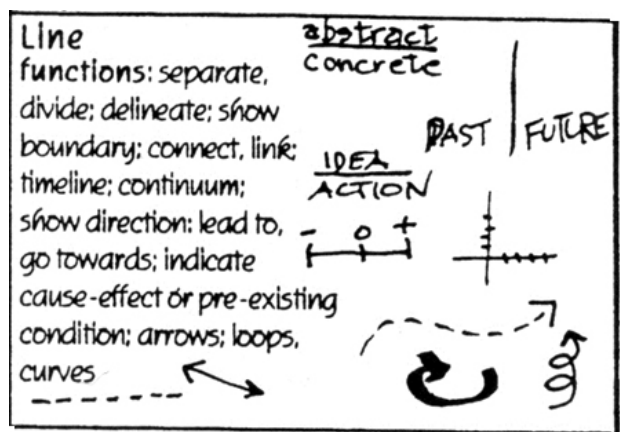

Fig 5. Linhas

Fonte: Hansen (1999)

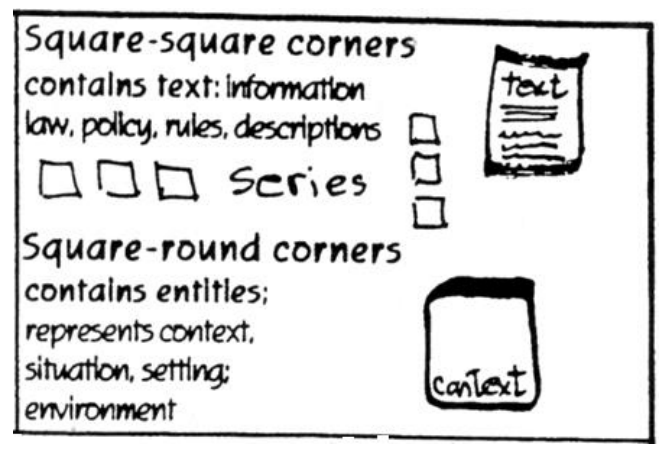

Fig 3. Áreas com ângulo reto ou com

cantos arredondados

Fonte: Hansen (1999)

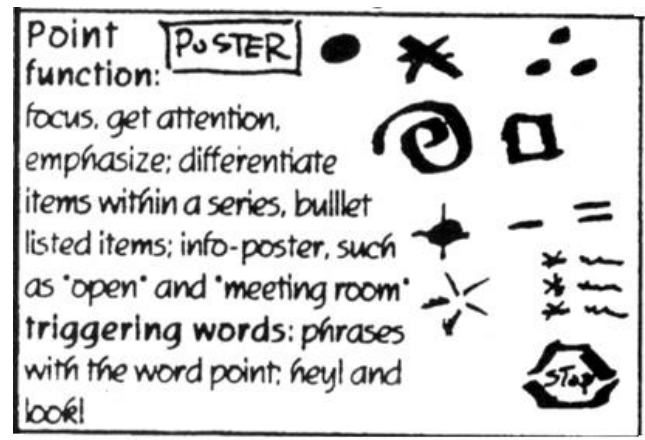

Fig 6. Pontos

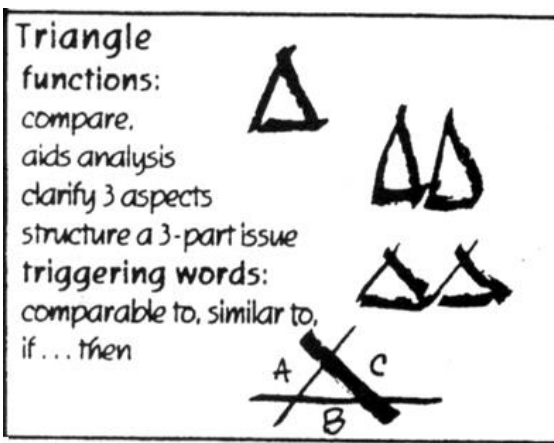

Fig 4. Triângulos

Fonte: Hansen (1999)

Fuzz or Fuzzy Idea functions: represent emerging notion, unformed thought, incomplete idea: issue needing clarification and structure; emerging intellectual material triggering words: confusing. unclear, muddled, complex

Fig 7. Rabiscos

Fonte: Hansen (1999)

Fonte: Hansen (1999)

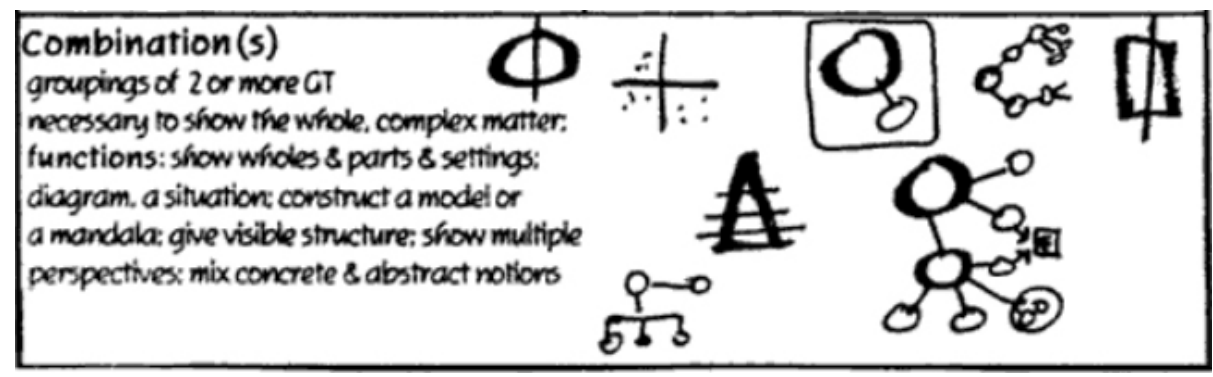

Fig 8. Combinação

Fonte: Hansen (1999)

Triângulos (figura 4) representam três aspectos de alguma coisa e são usados como ponto de partida para análise; para pensar; contrastar três aspectos de uma entidade (HANSEN, 1999).

As Linhas (figura 5) representam as conexões; ligação; direção; fronteira e separação e são usadas para conectar entidades, sistemas; para feedback ou direcionar caminhos e repetições; separar; dividir e enfatizar. Por outro lado, os Pontos (figura 6) representam um ponto no tempo e são usados para focar; chamar atenção; fazer um ponto; enfatizar e listas itens (HANSEN, 1999). 
Os Rabiscos (figura 7) representam o desconhecido; o não rotulado; conceito novo; ideia ou noção; entidade não desenvolvida ou não explorada; o obscuro; o confuso; e podem ser usados como uma marca para entidade desconhecida ou emergente para ser clarificada (HANSEN, 1999).

Por outro lado, a Combinação (figura 8) representa entidades complexas; problemas de muitas partes; estágios ou fases; evolução; mudança da natureza do problema através do tempo; múltiplas perspectivas; diferindo pontos de vista; diferentes relacionamentos; abstrações concretizadas. Desta forma, cada uma das ferramentas gráficas de Hansen representa uma variedade de situações e pode ser usadas de diversas formas (HANSEN, 1999).

\section{Discussão sobre as Representações Gráficas do Projeto de Pesquisa}

O projeto de pesquisa representado tem por objetivo identificar as vantagens do uso de histórias em quadrinhos eletrônicas como meio publicitário em relação à percepção e memória dos usuários na divulgação de bens e serviços quando comparados com os banners tradicionais. Esta pesquisa está posicionada entre três áreas de conhecimento: Informática, Design e Publicidade, o que torna a pesquisa mais difícil de ser entendida e descrita.

A fim de esclarecer melhor as ideias que se tem deste projeto, recorre-se às representações gráficas descritas neste artigo. Espera-se que, tanto o mapeamento de informações de Horn quanto as ferramentas gráficas de Hansen auxiliem no entendimento do projeto como um todo e gerem conteúdo para a delimitação do projeto de pesquisa.

De acordo com Horn (1974), o mapeamento de informação é um sistema de princípios e procedimentos que tem como objetivo identificar, categorizar, inter-relacionar e sequenciar e apresentar graficamente a informação solicitada, além de ser um método de aprendizagem que reúne a investigação e a instrução no desenvolvimento de materiais para melhorar a apresentação da comunicação técnica.

A estruturação do documento em blocos de informações faz com que a leitura se torne agradável e direcionada através dos assuntos que interessam ao leitor. Ele pode buscar as informações necessárias em qualquer bloco de informação, sendo guiado pelos rótulos dos blocos que estão próximos do conteúdo aos quais se referem.

No mapa de informações, os textos apresentados são considerados essenciais para cada bloco relacionado. Desta forma, o leitor terá em mãos a essência do assunto, evitando que se perca tempo de leitura atrás do que realmente interessa a ele.

O projeto de pesquisa é representado na forma de um mapa de informação (figura 9). Nele são descritas as partes principais de um projeto de pesquisa. Ainda que seja apenas uma parte do projeto, percebe-se que o uso do mapa de informação realmente facilita a leitura do documento. 


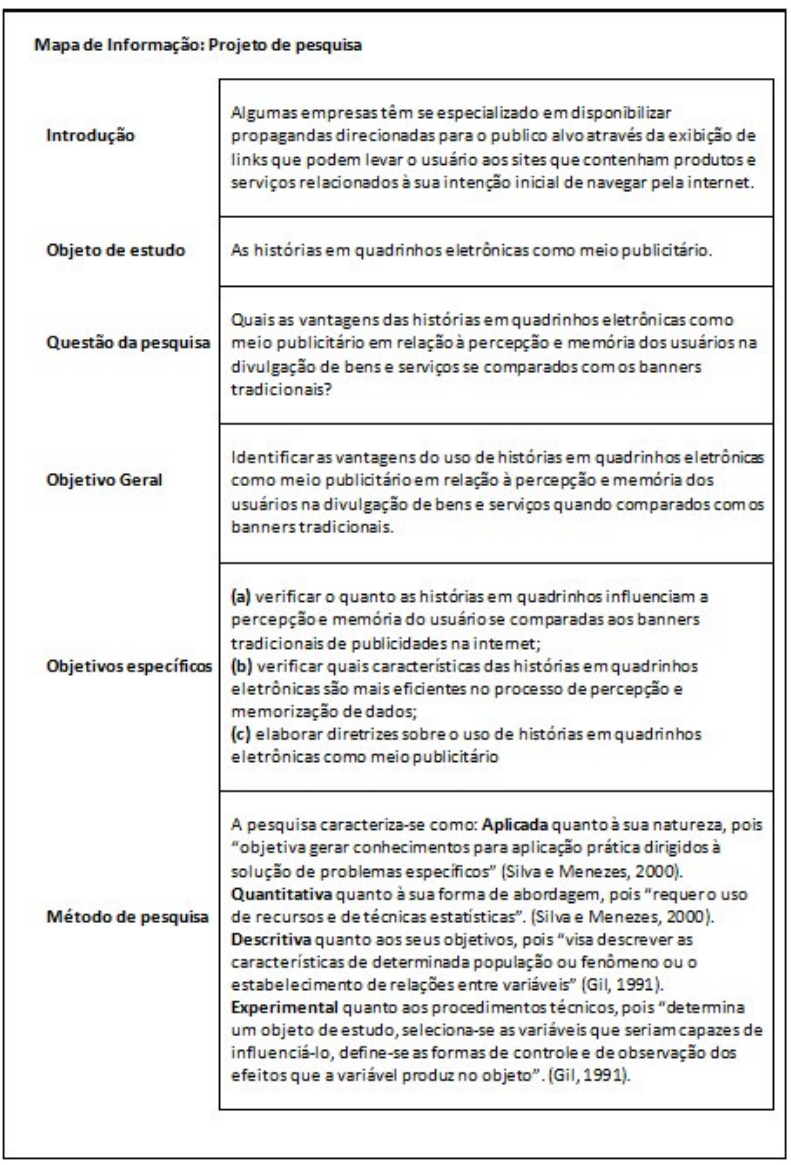

Na Figura 10, o mesmo projeto de pesquisa é representado. Desta vez usando as ferramentas gráficas de Hansen. Nem todas foram utilizadas, mas a combinação entre várias delas tornou a representação gráfica compreensível. O uso de textos explicativos auxilia no entendimento de conceitos e conteúdos que se deseja explicar.

Hansen (1999) defende que simples formas gráficas podem representar a cognição pré- verbal e dar forma concreta a ela. E isso é percebido na representação acima. Embora a leitura desta representação não seja tão fácil quanto à leitura dos mapas de informação, percebe-se sua relevância para que os conceitos sejam entendidos antes de serem escritos.

O uso de gráficos permitiu uma melhor visualização de todo o contexto da pesquisa, desde as áreas de conhecimento até os objetivos e metodologias e como eles se relacionam.

Fig 9. Representação gráfica de parte de um projeto de pesquisa usando um mapa de informação de Horn Fonte: $O$ autor (2017)

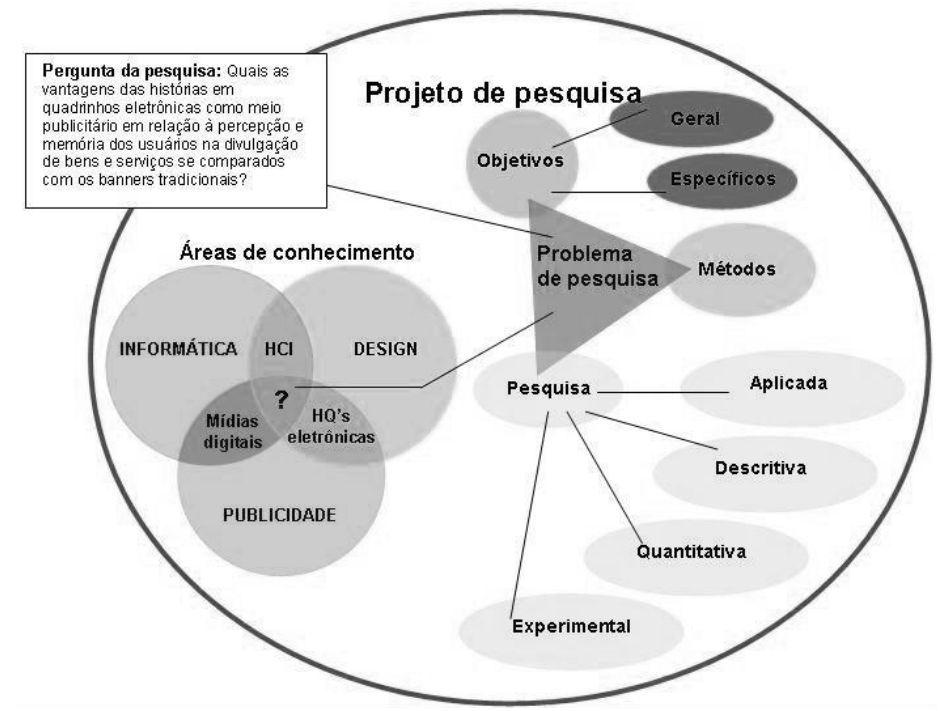

Fig 10. Representação gráfica de parte de um projeto de pesquisa usando um mapa de informação de Horn

Fonte: $\mathrm{O}$ autor (2017) 


\section{Conclusão}

Os estudos de Horn e de Hansen não são opostos entre si. Enquanto Horn pesquisou o uso de diagramas no desenvolvimento de documentos, Hansen estudou o uso dos diagramas como forma de pensar e expressar conceitos.

Enquanto Horn desenvolveu uma organização gráfica mais descritiva, Hansen optou pelo aproveitamento máximo de figuras e formas para esclarecer e expressar ideias. Não se pretendia comparar as duas abordagens e discutir a melhor em relação à outra.

Foi realizada uma revisão de literatura sobre o assunto e foram aplicadas as duas abordagens na solução de um mesmo problema, gerando dois resultados diferentes entre si, mas que se complementam.

A partir destes resultados, sugere-se que as ferramentas gráficas de Hansen sejam usadas no processo inicial de especificação de um problema. Suas ferramentas podem ser muito úteis para a clarificação de ideias, visualização de inter-relações e compreensão de conceitos e do funcionamento do problema em questão.

Percebe-se também que o mapeamento de informação não é tão eficiente na fase inicial do estudo do problema, período em que os conceitos estão nebulosos, mas quando ele já estiver mais bem definido. Percebe-se que os mapas de informação são muito mais úteis quando já se sabe o fazer e o que estudar, mas ainda precisa organizar as informações.

\section{Referências}

ARCHELA, RS, 1999. Imagem e representação gráfica. Geografia, Londrina, v. 8, n. 1, p. 5-11, jan. /jun.

GIL, A. C., 1999. Métodos e técnicas de pesquisa social, São Paulo: Atlas

Hansen, Y. M., 1999. Graphic tools for thinking, planning, and problem solving, in Information Design, ed. by Robert Jacobson, MIT Press.

Horn, R. E., 1974. Information Mapping, from Training in Business and Industry. Vol.11, No. 3. Horn, R. E. (1992). Clarifying Two Controversies about Information Mapping's Method. Educational and Training Technology International 29(2): 10917.

Horn, R. E., 1993. Structured writing at twenty-five, Performance and Instruction 32(February):11-17.

Horn, R. E., 1999. Two Approaches to Modularity: Comparing the STOP Approach with Structured Writing, Journal of Computer Documentation.

Silva, E. L; Menezes, E.M., 2000. Metodologia da pesquisa e elaboração de dissertação, Florianópolis: Laboratório de Ensino a Distância da UFSC. 